\title{
Fracture in Norway spruce wood treated with Physisporinus vitreus
}

\author{
Marjan Sedighi Gilani' ${ }^{1}$ Markus Heeb $^{2} \cdot$ Anja Huch $^{1}$ • \\ Siegfried Fink ${ }^{3}$ - Francis Willis Mathew Robert Schwarze ${ }^{2,3}$
}

Received: 19 January 2016/Published online: 6 October 2016

(C) Springer-Verlag Berlin Heidelberg 2016

\begin{abstract}
Changes in the fracture behaviour of Norway spruce tonewood after fungal treatment were studied. Specimens were incubated for 6, 9 and 12 months with Physisporinus (P.) vitreus. Fracture tests were performed in a compact-tension fracture experiment set-up, and the results were compared with the morphological analysis of the degraded wood structure and transverse sections of the crack tip viewed under light and fluorescence microscopy. It was evident that both the failure load and critical stress intensity factors were reduced in wood after prolonged incubation periods. Weight losses were significantly higher in sapwood than in heartwood. With prolonged incubation periods, the frequency of unstable fracture and brittle behaviour of the wood increased. In untreated wood, cracks were initiated in the earlywood. The process involved both delamination of the cells within the middle lamellae and rupture of the cell walls, inducing a zigzag crack tip pattern. In fungally treated wood, cracks often commenced from the intersection between late- and earlywood, resulting in a straight tangential crack line. Micrographic images showed that $P$. vitreus was more active in the secondary walls of latewood tracheids. In this region of the wood, the cell walls were strongly degraded after 9-12 months of incubation, resulting in a reduction in tensile strength, even though the wood did not show strong features of decay at the macroscopic level.
\end{abstract}

Marjan Sedighi Gilani

marjan.gilani@gmail.com

1 Applied Wood Material Laboratory, (EMPA) Swiss Federal Laboratories for Materials Science and Technology, 129 Uberlandstrasse, 8600 Dübendorf, Switzerland

2 Applied Wood Material Laboratory, (EMPA) Swiss Federal Laboratories for Materials Science and Technology, Lerchenfeldstrasse 5, 9014 St-Gallen, Switzerland

3 Professur für Forstbotanik, Albert-Ludwigs-Universität Freiburg, Bertoldstrasse 17, 79085 Freiburg Im Breisgau, Germany 


\section{Introduction}

After infection, colonization and if conducive conditions permit, wood decay fungi can significantly alter the mechanical properties of wood. The extent of damage depends highly on the wood structure, decay type, lignin composition, fungal species, susceptibility and duration of wood exposure to degradation. Several studies focus on analysis of changes in cellulose, hemicellulose and lignin constituents in wood (Pandey and Pitman 2003; Ray et al. 2005; Schwarze 2007; Lehringer et al. 2010). White rot fungi degrade different wood components, i.e. cellulose, hemicellulose and lignin at different rates. Interestingly, the white rot fungus Physisporinus vitreus degrades the sapwood at higher rates than the heartwood. Lehringer et al. (2011) showed that in sapwood the white rot fungi, for example $P$. vitreus, degrade cellulose more extensively during early stages of degradation, whereas in the heartwood cellulose and lignin are degraded almost proportionally to another. The preferential degradation of bordered pit membranes in Norway spruce wood and the selective delignification of the secondary wall of late wood tracheids by the fungus have been used for biotechnological engineering and wood modification processes, for example enhancing the uptake of preservatives in refractory wood (Schwarze et al. 2006) or for improving the vibrational properties of tonewood for wood of violins (Schwarze et al. 2008; Sedighi Gilani et al. 2014a, b, 2016).

During longer incubation periods, the removal of cell wall constituents from the wood may result in a strong reduction in the mechanical properties in bulk material (Maeda et al. 2015). In addition, wood decay increases the potential of the development of 'wet pockets' phenomena that occur mainly in hardwoods, but also in softwoods such as Norway spruce wood (Smith et al. 2003). Wet pockets are localized areas that are as wide as few growth rings within the boundary of sap- and heartwood. These regions show high moisture content and facilitate initiation of mechanical damage and fracture. Crack propagation and fracture are also influenced by the heterogeneous distribution of the growth layers across a wood section and early- and latewood tracheids with different microstructure and stiffness. While the relation between fracture behaviour of wood and microstructure and alternation of early- and latewood cell regions have been widely discussed (Boatright and Garrett 1983; Dill-Langer et al. 2002; Landis et al. 2002; Sedighi Gilani and Navi 2007), the role of microstructural damage, for example due to fungal decay, has received less attention.

Previous studies reported on changes in the vibro-mechanical properties of tonewood modified with $P$. vitreus, for application to string instruments. It was shown that the modification process improves the internal damping, sound radiation and acoustic impedance (Sedighi Gilani et al. 2014a, b, 2016), all of which have a positive influence on the acoustic properties of string instruments. However, the specific modulus of elasticity is reduced up to $10 \%$ in both the parallel and perpendicular directions to the grain in wood incubated with $P$. vitreus. Reduction in the mechanical properties may result in the development of microcracks which subsequently will impair the acoustic properties of tonewood for music instruments. 
The objective of this study was to investigate the fracture behaviour of wood after incubation with $P$. vitreus and to discuss the implications for developing a standardized treatment method. Fracture tests were performed in the RL direction in a mode I compact-tension set-up on untreated (control) and fungally treated Norway spruce wood specimens after 6, 9 and 12 months of incubation. Specimens were conditioned at $50 \%$, and the tests were performed at the same relative humidity, to avoid the influence of moisture content change on fracture properties (Vasic and Stanzl-Tschegg 2006). Mode I fracture corresponds to the opening mode, while R index shows the direction perpendicular to the grain of the crack, and $\mathrm{L}$ index specifies the direction of crack extension. RL is often the primary focus of fracture studies in wood, similar to natural cleavage planes from which cracks can easily propagate (Smith et al. 2003).

\section{Materials and methods}

\section{Preparation of samples and fungal treatment}

Specimens were cut from four tonewood planks of Norway spruce wood (Picea abies L.) from a tree felled in autumn 2009 in the Surfers region, Switzerland. All planks had dimensions of 50 (L: longitudinal $) \times 16(\mathrm{R}$ : radial $) \times 4(\mathrm{~T}$ : tangential $)$ $\mathrm{cm}^{3}$ and were excised as twin specimens along the longitudinal direction. They had narrow and even annual rings and were perfectly quartered with minimal run out according to the criteria for 'master grade' tonewood used for string instruments craftsmanship. While one wood plank was used for controls, the other 3 were incubated in the dark at $22{ }^{\circ} \mathrm{C}$ and $70 \%$ relative humidity (RH), with $P$. vitreus, according to EN 113 (European Standard EN 113 1997). The incubation process was ended by cleaning and sterilizing the planks with ethylene oxide after 6,9 and 12 months of incubation. In Fig. 1a, images of a tonewood plank of Norway spruce, before and after incubation with $P$. vitreus and processing into two quarter cuts, are shown. From each plank, 24 specimens (12 from sapwood and 12 from heartwood) were cut for compact-tension fracture tests. In Fig. 1b, dimensions of the specimens and in Fig. 1c, two test specimens from the sap- and heartwood regions of the wood incubated for 9 months are shown. Instead of a sharp crack tip, the end of each notch was cut in a round shape to distribute the stresses more homogeneously in a wood region which included both early- and latewood cells. Thus, the crack was not forced to start from the notch tip, but could be initiated and propagate in the weakest regions, depending on the properties of early- and latewood cells and their bounding medium, before and after fungi treatment. Specimens were conditioned at a RH of $50 \%$ and temperature of $20{ }^{\circ} \mathrm{C}$ for 4 weeks, before and during the fracture tests.

\section{Fracture test}

Ninety-six compact-tension (CT) fracture tests were performed in total, 12 from the sap- and 12 from the heartwood of each tonewood plank. The tests were carried out in a Zwick 1474, with a maximum load capacity of 1000 N. For statistical analysis 
(a)

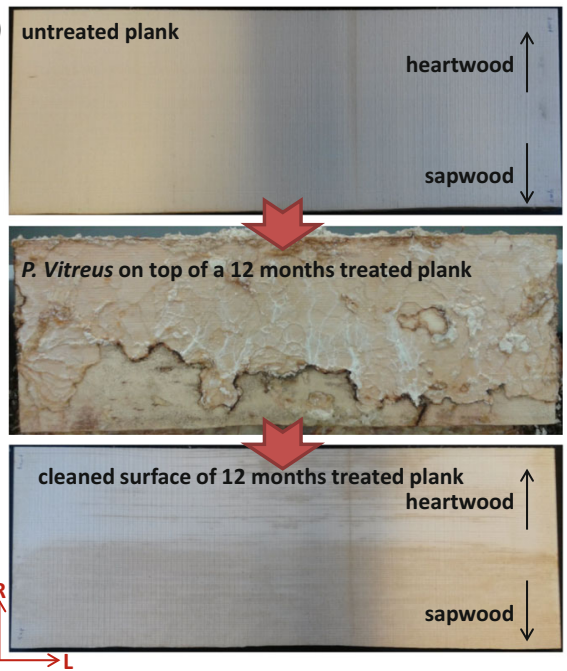

(b)

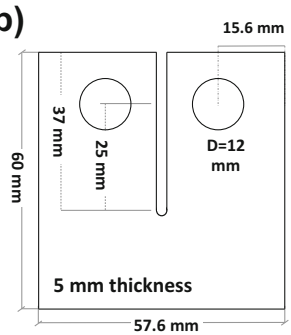

(c)

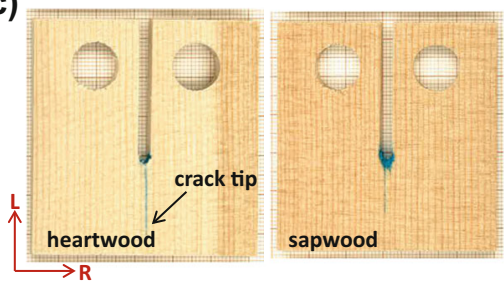

Fig. 1 a Top image of the untreated tonewood prior to incubation. Centre tonewood after incubation showing that the surface is completely colonized by mycelium. Bottom tonewood after being cleaned and cut, b geometry of excised specimens for compact-tension fracture test, c one sap- and heartwood specimen incubated for 9 months with $P$. vitreus after testing (crack tip in wood specimens is stained with Astra blue dye)

of the measured and calculated data, one-tailed significance test in Microsoft Excel 2010 was performed. Each specimen was loaded in tension, by pulling the pins which were gripped inside the wood holes, with a loading rate of $0.5 \mathrm{~mm} / \mathrm{min}$. The stress intensity factor $K_{I}$ was calculated from:

$$
K_{I}=\frac{F}{B \cdot \sqrt{w}} f\left(\frac{a}{w}\right)
$$

where $F$ is the applied load, $a$ is the opening length at the beginning of the test, $B$ is the specimen thickness, $W$ is the specimens width and $f(a / w)$ is a geometry constant calculated from (Bower 2009; Keunecke et al. 2012):

$$
f\left(\frac{a}{w}\right)=\left(2+\frac{a}{w}\right) \cdot \frac{0.886+4.64\left(\frac{a}{w}\right)-13.32\left(\frac{a}{w}\right)^{2}+14.72\left(\frac{a}{w}\right)^{3}-5.6\left(\frac{a}{w}\right)^{4}}{\left(1-\frac{a}{w}\right)^{1.5}}
$$

Critical stress intensity factor or fracture toughness, $K_{I c}$, was obtained by considering the critical force, $F_{\text {critical }}$, in Eq. 1. Strain at failure was calculated from dividing the deformation which leads to $F_{\text {critical }}$, by the width of the specimen. In addition, the specific fracture energy, $G_{f}$ was calculated from the area under the force-displacement curves, divided by the fracture section area. $K_{I c}$ is a property which describes the ability of material to resist the fracture, while $G_{f}$ relates to the total energy consumed by crack initiation and crack propagation. 


\section{Microscopy}

For general observation of wood anatomy and histology, test specimens were cut into smaller sizes of $5 \times 5 \times 5 \mathrm{~mm}^{3}$. They were fixed in 2 vol. $\%$ glutaraldehyde buffered at $\mathrm{pH}$ 7.2-7.4, dehydrated with isopropanol and embedded in a methacrylate medium, with the transverse face exposed for examination. The embedded specimens were sectioned at approx. 2 and $4 \mu \mathrm{m}$ thickness using a rotary microtome (Leica1 2040 Supercut) fitted with a diamond knife. The unstained sections were mounted in glycerine and examined for autofluorescence with a Zeiss ${ }^{\circledR}$ fluorescence microscope, using incident illumination from a mercury vapour lamp with a filter combination (BP 365, FT 395 and LP 397).

Further, to analyse the crack tip in a larger field of view, drops of diluted Astra blue dye were dropped on top of each crack notch, and thus, the close proximity to the crack tip was marked by the penetration of blue colour inside the crack. Afterwards, specimens of $5 \times 5 \times 5 \mathrm{~mm}^{3}$ from crack vicinity were cut and embedded in epoxy (Araldite) and polished. Images were acquired using a digital microscope (Leica DVM2500 with LAS software and a CCD camera, Leica Microsystem) at low magnification.

\section{Results and discussion}

Gravimetry of wood planks at $50 \% \mathrm{RH}$ showed $2.4,2.6$ and $3.3 \%$ weight losses after 6, 9 and 12 months of incubation, respectively. In Fig. 2, the forcedisplacement responses of the untreated (control) and sapwood treated for 6, 9 and 12 months are compared. The first phase of the force-displacement curve shows the linear elastic response of the material, with slope of $\alpha$, which is proportional to wood stiffness in the radial direction. The first visible crack often occurred at the peak load. However, development of microcracks is likely to be initiated at the critical force, when the slope of the linear part begins to decline. After the peak, the measured force was reduced, while the crack propagated through the specimen. In the control samples, the material behaviour after peak was stable in some of the specimens and unstable in the others, i.e. a sudden reduction in force was measured after the development of first visible crack. The material softening was more likely stable in the wood treated for 6 months. On the contrary, a large number of specimens showed a brittle behaviour, after 9 and 12 months of treatment, with a peak force occurring at half of the other test specimens. In Table 1, the average density, $\rho$, slope of the force-displacement curves, $\alpha$, critical force, $F_{\text {critical }}$, critical stress intensity factor, $K_{I c}$, and fracture energy, $G_{f}$, of each group of specimens are presented. $\rho$ in the heartwood was smaller than in the sapwood, both in control and in the treated wood. This correlates with the fact that in the sapwood of the selected planks, the growth rings were significantly narrower than in the heartwood, and thus, a larger fraction of latewood rings (with higher density) was present in the same volume of material compared to the heartwood. In addition, $\alpha, F_{\text {critical }}, K_{I c}$ and $G_{f}$ were higher in the sapwood when compared to heartwood in the untreated specimens. After treatment, this trend changed and the sapwood showed inferior 
(a)

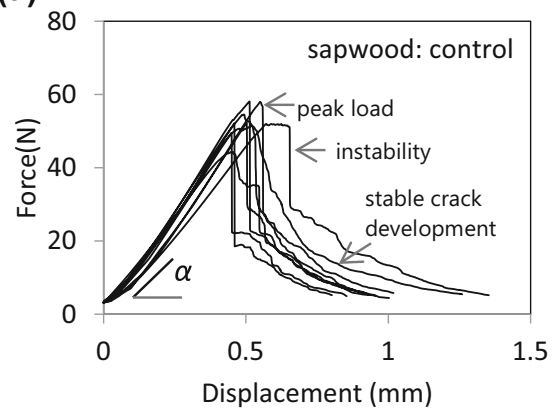

(c)

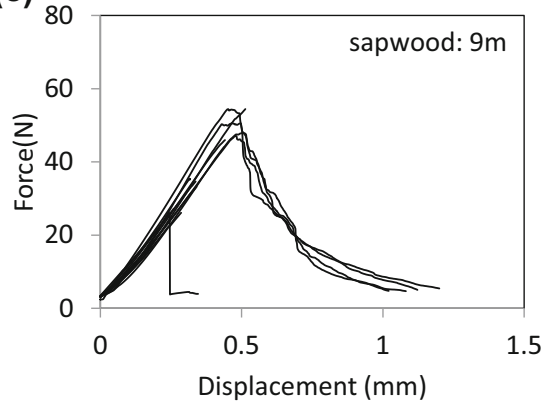

(b)

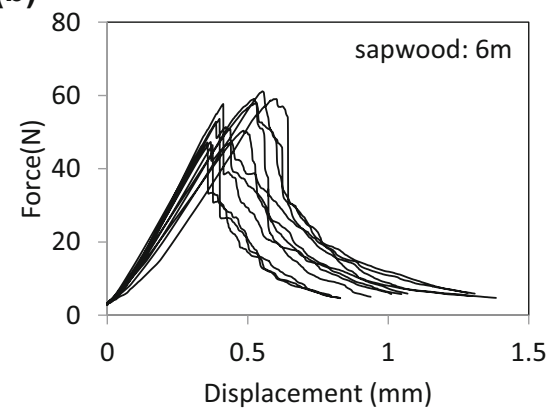

(d)

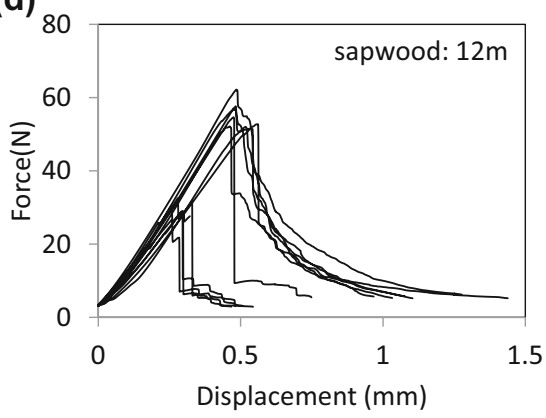

Fig. 2 Force-displacement curves of a untreated, b 6 months incubated, c 9 months incubated and d 12 months incubated sapwood

mechanical properties. Reduction in the mechanical properties in sapwood indicates that the sapwood was more susceptible to decay than the heartwood. This observation and also the more significant change of the wood colour in the sapwood than in the heartwood (also observed in Fig. 1) are in good agreement with the literature (Lehringer et al. 2011).

$K_{I c}$ measures the ability of the material to resist the crack propagation, which was significantly reduced in the sapwood after fungal treatment. According to twosample $t$ test (assuming equal variances), confident level for this observation was $98.4 \%$ after 6 months and $99.9 \%$ after 9 and 12 months of treatment. Also $G_{f}$, which is not dependent on the precise crack tip geometry like $K_{I c}$, was reduced after treatment, with confident level of $78 \%$ after 6 months of treatment, $73 \%$ after 9 months and $96.8 \%$ after 12 months. However, no significant trend in terms of reduction was observed for $K_{I c}$ and $G_{f}$ in the heartwood after incubation. Scattering between the results in heartwood was more likely related to natural heterogeneities in the wood, while the specimen batches for control and the treated wood were cut from the same tree but at 4 different heights. In Fig. $3, F_{\text {critical }}, K_{I c}, G_{f}$ and also the maximum strain at failure, $\varepsilon_{\text {failure }}$, of all sapwood specimens are compared in a boxand-whisker plot presentation. This presentation shows the possibility of skewed distribution of the data, which indicates that the mean and standard deviation in Table 1 might not be a full comprehensive measure of the change in material 


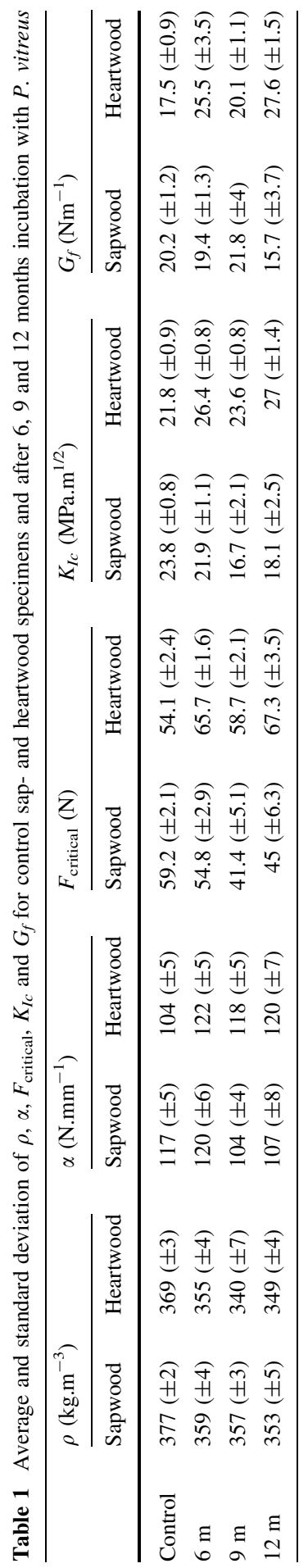


(a)

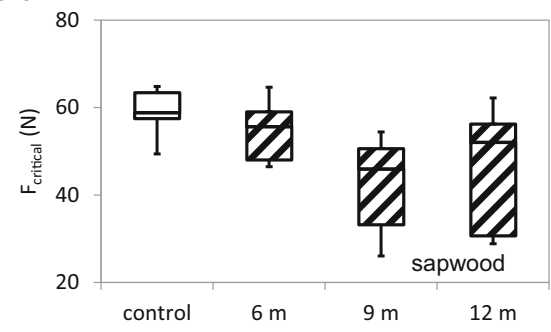

(c)

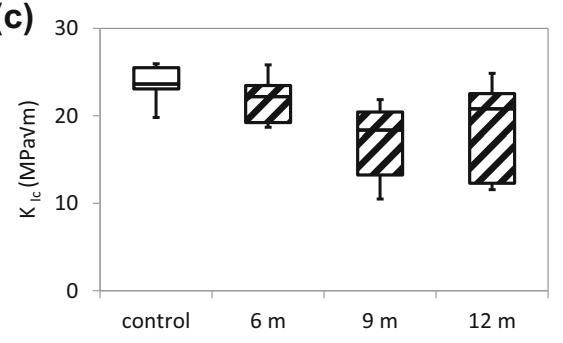

(b)

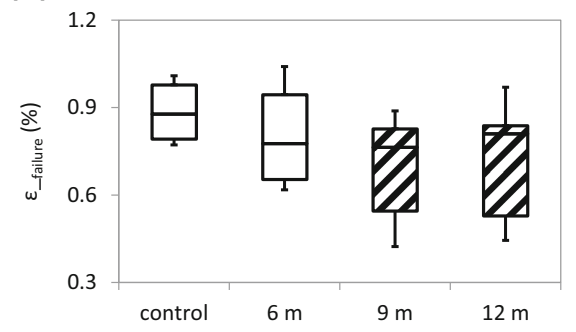

(d)

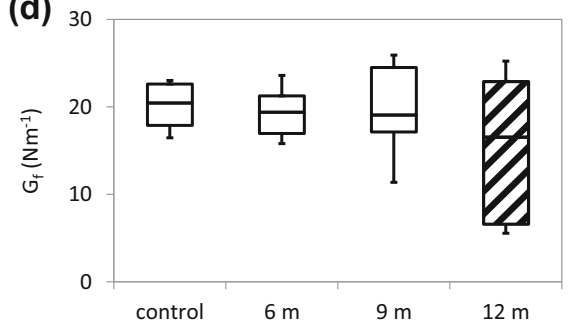

Fig. 3 Box-and-whisker plots of a critical force, $\mathbf{b}$ strain to failure, $\mathbf{c}$ critical stress intensity factor and d specific fracture energy at different states of fungal decay sapwood. All groups of data which are significantly less than their control values (assumed as $P$ value $\leq 0.05$ ) are pattern-filled

property after fungal treatment. Nevertheless, the reducing trend of the mechanical properties in fungally treated wood was clear, especially at longer incubation periods. The pattern-filled box plots highlight when the measured or calculated properties of the modified wood were significantly lower $(P$ value $\leq 0.05)$ than the control specimens. Moreover, the scattering between the data was more significant after fungal incubation, as illustrated by the larger differences between the first and third quartiles of fungally treated wood in Fig. 3b. This is mainly due to the more brittle nature of failure in fungally treated wood. As a result, some of the specimens endured a similar critical force to that in the untreated wood and some others failed at much lower force and in a brittle manner.

Scattering of the data after fungal treatment is attributed to the inhomogeneity of degradation in the wood microstructure. In Fig. 4, micrographs of wood incubated for 6, 9 and 12 months under the fluorescence microscope are illustrated. At 6 months, slight degradation of secondary walls started and is highlighted with white pointers. The micrographs also show the advanced degradation of the tracheid cell walls after 9-12 months of incubation with $P$. vitreus and initialisation of the first cracks. At advanced stages, the secondary walls of latewood tracheids were significantly thinned, and at the location of bordered pits, the tangential cell walls were locally destroyed. Degradation was less extensive in the earlywood, with discrete thinning of the secondary walls and preferential degradation of bordered pit membranes. In addition, the compound middle lamella was not significantly degraded. As this layer is responsible for bonding of the cell walls of two adjoining tracheids together, its integrity helps wood to partially preserve the transversal 
Fig. 4 Transverse section of incubated Norway spruce wood with $P$. vitreus, viewed under the fluorescence microscope after a 6 months, b 9 months and c 12 months. Apparent thinning of the secondary walls are highlighted with pointers, while asterisks show the strong degradation of tangential cell walls at the intersection between early- and latewood
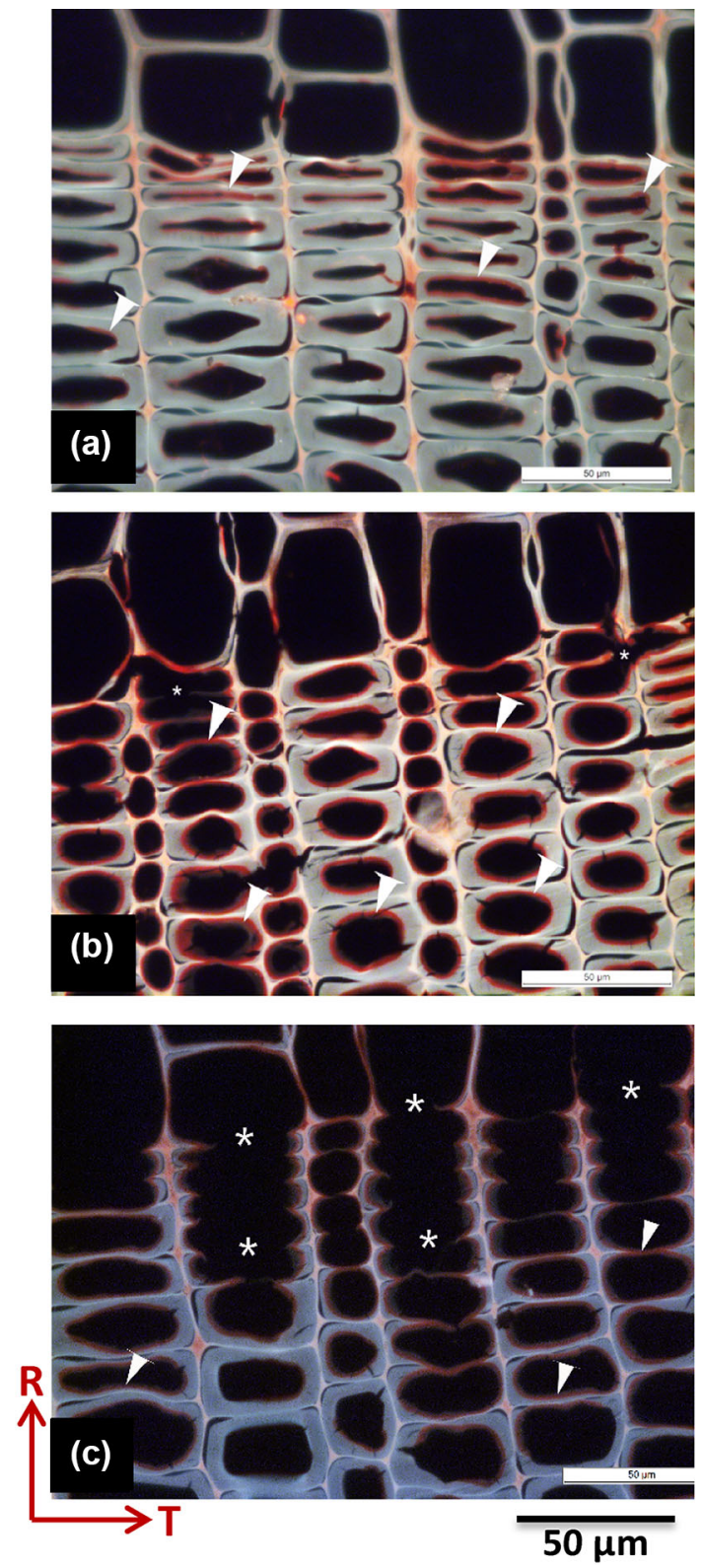

stiffness and also thoroughness in perpendicular to the grain direction, even after 12 months of incubation. However, localized degradation of the latewood tracheids created weak areas in the wood structure, especially at the interface between latewood and earlywood tracheids. This resulted in a brittle fracture of the test specimens, especially in the incubated sapwood after longer incubation periods. This effect is evident in Fig. 5, which shows the crack tip of a control and a 

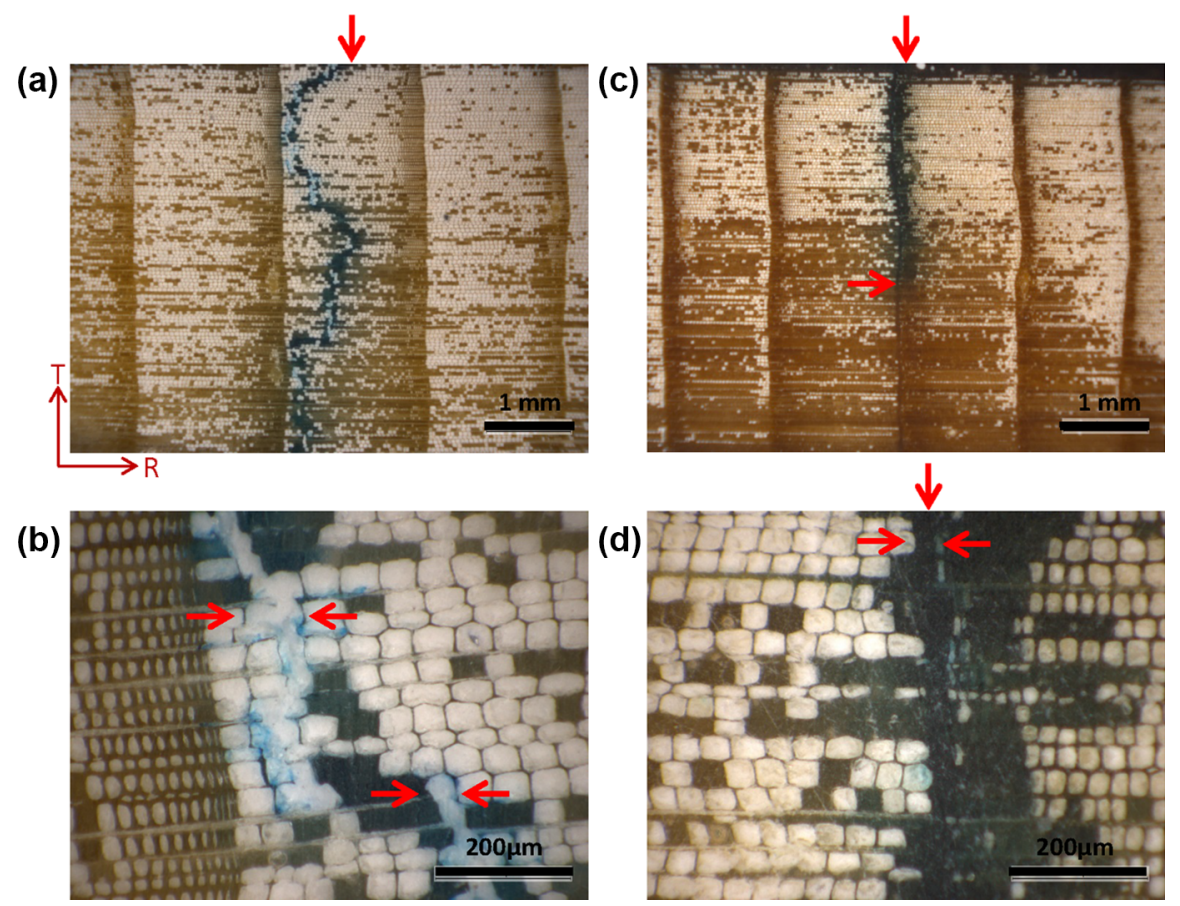

Fig. 5 Morphology of the crack tip at two different magnifications, in $\mathbf{a}$ and $\mathbf{b}$ control Norway spruce, and $\mathbf{c}$ and $\mathbf{d}$ after 12 months incubation

12 months treated wood specimen over the entire specimen thickness. Two principle fracture mechanisms were identified which were affected by the integrity of the wood microstructure. The first failure mechanism observed, mainly in untreated wood, was a zigzag crack line in the earlywood (Fig. 5a). This crack morphology provides evidence for a crossover bridging of the wood cells behind the crack tip, which contributes significantly to fracture toughness (Gamstedt et al. 2013). Figure $5 \mathrm{~b}$ illustrates the rupture of the secondary walls. The other mechanism formed a tangential, straight crack line in fungally treated wood (Fig. 5c), in the latewood or at the interface of early- and latewood in the highly degraded wood, as a result of a brittle failure. At the cell wall level, the process involved both delamination of the cells at the middle lamellae and rupture of the degraded cell walls.

\section{Conclusion}

The fracture behaviour of Norway spruce tonewood specimens, before and after incubation with $P$. vitreus for 6, 9 and 12 months, was studied. In untreated wood, the sapwood showed superior mechanical properties compared to the heartwood. After fungal incubation, the critical force, fracture toughness and stress intensity 
factor were significantly reduced in sapwood with increasing incubation periods, while the measured differences in the heartwood properties were more likely related to inhomogeneity of the wood itself. Reduction in the strain at failure and also the material toughness showed that the sapwood was rendered more brittle after fungal incubation. In addition, scattering between results showed that degradation of the sapwood was not uniform in all specimens, i.e. some parts of the wood were less affected than others. $P$. vitreus preferentially degrades the secondary walls of the latewood tracheids by progressive cell wall thinning. After 12 months of incubation and in the presence of bordered pit membranes, the tangential cell walls were locally completely destroyed. In the earlywood, degradation occurred discretely in the cell walls and pit membranes. The extent of degradation in the compound middle lamella was not significant. While according to previous studies, incubation improves the resonance properties of tonewood for instrument making, the present study showed that the time of incubation is a critical limiting factor in terms of fracture and stability of the material during crafting of the instrument and its longevity.

Acknowledgments We would like to acknowledge the financial support of the Walter Fischli Foundation. We also acknowledge the contribution of Javier Ribera for support on experiments with fungi, Daniel Herr for rigorous sample preparation at EMPA wood workshop and Walter Risi for expertise in fracture test set-up.

\section{References}

Boatright SWJ, Garrett GG (1983) The effect of microstructure and stress state on the fracture behaviour of wood. J Mater Sci 18:2181-2199

Bower AF (2009) Applied mechanics of solids. CRC Press, Boca Raton

Dill-Langer G, Lutye S, Aicher S (2002) Microfracture in wood monitored by confocal laser scanning microscopy. Wood Sci Technol 36:487-499

European Standard EN 113 (1997) Wood preservatives: test method for determining the protective effectiveness against wood destroying basidiomycetes. Determination of toxic values. European Committee for Standardization, Brussels

Gamstedt EK, Bader TK, de Borst K (2013) Mixed numerical-experimental methods in wood micromechanics. Wood Sci Technol 47:183-202

Keunecke D, Niemz P, Tollert M, Hänsel A (2012) Mechanical properties and failure of yew and spruce determined with a compact tension test and digital image correlation. Wood Res 54:669-676

Landis EN, Vasic S, Davids WG, Parrod P (2002) Coupled experiments and simulations of microstructural damage in wood. Exp Mech 42:389-394

Lehringer C, Hillebrand K, Richter K, Arnold M, Schwarze FWMR, Militz H (2010) Anatomy of bioincised Norway spruce wood. Int Biodeterior Biodegrad 64:346-355

Lehringer C, Saake B, Živkovic V, Richter K, Militz H (2011) Effect of Physisporinus vitreus on wood properties of Norway spruce. Part 2: aspects of microtensile strength and chemical changes. Holzforschung 65:721-727

Maeda K, Ohta M, Momohara I (2015) Relationship between the mass profile and the strength property profile of decayed wood. Wood Sci Technol 49:331-344

Pandey KK, Pitman AJ (2003) FTIR studies of the changes in wood chemistry following decay by brownrot and white-rot fungi. Int Biodeterior Biodegrad 52:151-160

Ray M, Kleist G, Murphy R (2005) Decay assessment in a hardwood handrail at the South Bank, London. J Inst Wood Sci 17(1):51-58

Schwarze FWMR (2007) Wood decay under the microscope. Fungal Biol Rev 21:133-170 
Schwarze FWMR, Landmesser L, Zgraggen B, Heeb M (2006) Permeability changes in heartwood of Picea abies and Abies alba induced by incubation with Physisporinus vitreus. Holzforschung 60:450-454

Schwarze FWMR, Spycher M, Fink S (2008) Superior wood for violins wood decay fungi as a substitute for cold climate. New Phytol 179:1095-1104

Sedighi Gilani M, Navi P (2007) Micromechanical approach to wood fracture by three-dimensional mixed lattice-continuum model at fiber level. Wood Sci Technol 41(7):619-634

Sedighi Gilani M, Tingout P, Heeb M, Schwarze FWMR (2014a) Influence of moisture on the vibromechanical properties of bio-engineered wood. J Mater Sci 49(22):7679-7687

Sedighi Gilani M, Boone MN, Mader M, Schwarze FWMR (2014b) Synchrotron X-ray microtomography imaging and analysis of wood degraded by Physisporinus vitreus and Xylaria longipes. Struct Biol 187:149-157

Sedighi Gilani M, Neuenschwander J, Heeb M, Furrer R, Sanabria SJ, Stoel BC, Schwarze FWMR (2016) Influence of incubation time on the vibration and mechanic properties of mycowood. Holzforschung 70(6):557-565

Smith I, Landis E, Gong M (2003) Fracture and fatigue in wood. Wiley, Chichester. ISBN 978-0-47148708-1

Vasic S, Stanzl-Tschegg S (2006) Experimental and numerical investigation of wood fracture mechanisms at different humidity levels. Holzforschung 61:367-374 